\title{
Structural phase transitions and piezoelectric anomalies in ordered $\mathrm{Sc}_{0.5} \mathrm{Ga}_{0.5} \mathrm{~N}$ alloys
}

\author{
A M ALSAAD and A A AHMAD* \\ Department of Physical Sciences, Jordan University of Science \& Technology, P.O. Box 3030, Irbid 221100, Jordan
}

MS received 5 February 2007

\begin{abstract}
Local-density approximation calculations (LDA) within density functional theory (DFT) and Berry phase approach within modern theory of polarization are performed to predict the structural and piezoelectric properties of ordered $\mathrm{Sc}_{0.5} \mathbf{G a}_{0.5} \mathrm{~N}$ alloys under compressive and tensile in-plane strain. This alloy is found to exhibit a tremendous piezoelectric response, associated with a phase transition from nonpolar $p 6_{3} / \mathrm{mcc}\left(D_{6 \mathrm{~h}}\right)$ space group to a polar $p 6_{3} m c\left(C_{6 v}\right)$ structure, at fixed $\mathrm{Ga}$ and $\mathrm{Sc}$ compositions when continuously changing the experimental accessible parameters (i.e. the compressive and tensile strain). The mechanism of the effects behind such anomalous behaviour is revealed and explained.
\end{abstract}

Keywords. Phase transitions; piezoelectric coefficients; ordered $\mathrm{Sc}_{0.5} \mathrm{Ga}_{0.5} \mathrm{~N}$ alloys.

\section{Introduction}

The optical, piezoelectric and dielectric properties of III$\mathrm{N}-\mathrm{V}$ semiconductors are of crucial importance, for instance, the change in the size of GaN quantum dots leads to photoluminescence in the entire visible spectrum (Damilano et al 1999; Edgar et al 1999) and thus of great interest for the light-emitting devices (LEDs). The GaN, InN and AlN have been the focus of intensive research for physical and technological reasons. The ability to form alloys and heterostructures of these compounds with continuously varying band gap led to the fabrication of optoelectronic devices such as light-emitting diodes, laser diodes and detectors with invaluable technological applications. Unlike the non-polar cubical conventional III-V semiconductors (such as GaAs, InAs, or AlAs), nitride binaries stabilize in the so-called wurtzite structure with the space group $\mathrm{Pb}_{3} m c$, which is polar (i.e. exhibits spontaneous polarization) and four-fold coordinated (Limpijumnog and Lambrecht 2001). Furthermore, due to the exceptional small atomic volumes of nitride semiconductors in comparison with other conventional III-V semiconductors, they exhibit larger optical band-gap (e.g. $E_{\mathrm{g}} \approx 3.5 \mathrm{eV}$ in $\mathrm{GaN}$ ), high thermal conductivity (see Edgar et al 1999 and references therein), which make these semiconductors good candidates for short-wave, high power photoluminescence devices (Christensen and Gorczyca 1994; Damilano et al 1999). However, the hightemperature applications of these devices require the replacement of InN (due to its low melting point) with another

\footnotetext{
*Author for correspondence (sema@just.edu.jo)
}

compound which has thermal properties suitable to be used in these devices. It has been indicated (Dismukes and Moustakas 1996) that ScN, because of its high melting temperature and lattice spacing match with $\mathrm{GaN}$, may be the right choice for the replacement of InN.

Recently, ScN has attracted rather more attention. Travaglini et al (1986) performed optical reflectivity measurements on practically stoichiometric single crystals of $\mathrm{ScN}$ and concluded that it is a compensated semimetal. More recently, experimental and ab initio computational methods have been employed to conclusively show that $\mathrm{ScN}$ is a semiconductor rather than a semimetal, i.e. there is a gap between the $\mathrm{N} 2 p$ and the Sc $3 d$ bands (Lambrecht 2000; Gall et al 2001). A pioneering study (Takeuchi 2002) using first-principles total-energy calculations to investigate the structural and electronic properties of $\mathrm{ScN}$ in all possible crystallographic phases has predicted that rocksalt is the most stable ground-state structure for $\mathrm{ScN}$, and since other group IIIA nitrides crystallize in the wurtzite structure, the fabrication of Sc-IIIA-N alloys becomes possible and might play a critical role in the future of 'smart' multifunctional devices operating at high temperatures and covering a very broad spectral region ranging from near-infrared to near ultra-violet. Farrer and Bellaiche (2002) reported the discovery of an intermediate structure between wurtzite and rocksalt that was denoted as $h-\mathrm{ScN}$ and which is similar to the phase found in $\mathrm{MgO}$ and called $h-\mathrm{MgO}$ (Limpijumnog and Lambrecht 2001). This meta-stable phase is obtained by the relaxation of the internal structural parameter, $u$, and the axial ratio, $c / a$, while the hexagonal Bravais lattice of wurtzite is preserved (Limpijumnog and Lambrecht 2001). In this phase, which could exist in other compounds, the Sc atoms 
form bonds with nitrogen layers above and below it in an equal manner and the buckled layer of wurtzite becomes flat and the structure phase transforms from four-fold coordinated to a five-fold coordinated structure. The discovery of this meta-stable wurtzite phase in $\mathrm{ScN}$, which exhibits a band gap smaller than that of wurtzite $\mathrm{GaN}$, indicates the possibility of wurtzite disordered ScGaN alloys with varying band gap and unusual electronic and piezoelectric properties. These experimental and theoretical observations have encouraged the researchers to go beyond the study of the equilibrium structure of $\mathrm{ScN}$ to investigate the compressively strained hexagonal $h-\mathrm{ScN}$. Ranjan et al (2003) found that this fascinating compound exhibits several unusual structural, piezoelectric and optical properties when it is compressively strained in the basal plane. For instance, this study demonstrates the existence of a phase transition from non-polar to polar structure at a compressive in-plane phase close to $3.4 \%$, at which a huge enhancement of piezoelectricity occurs. In particular, the study predicted an $e_{33}$ piezoelectric coefficient of $12 \mathrm{C} / \mathrm{m}^{2}$, which is one of the largest piezoelectric coefficients ever predicted using first principles calculations and is even larger than the corresponding coefficients of ferroelectric perovskites ranging between $\approx 4$ and $9 \mathrm{C} / \mathrm{m}^{2}$.

A more recent experimental study, using rf molecular beam epitaxy, has evidently supported the latest theoretical predictions of the existence of the meta-stable layered $h$ $\mathrm{ScN}$ phase (Constantin et al 2004). This specific study has also shown that during the alloy formation in ScGaN, the existence of three distinguishable growth regimes over the Sc composition ranging between $x=0$ and $x=100 \%$, depends on the Sc composition. Moreover, the relative stability of different crystallographic phases and the possible strain-induced and high-pressure phase transformations among them have recently attracted a lot of attention in III-V semiconductors, in general and in III-V nitrides, in particular. Generally, one can think of two main types of bonded structures in III-V semiconductors: the four-fold coordinated tetrahedral structures, such as zinc blende (ZB) and wurtzite (WZ), and the octahedral structures, such as rocksalt (RS). The real ionic semiconductors (for example, $\mathrm{NaCl}, \mathrm{MgO}$ ) favour high coordination and thus prefer rocksalt for stability even at ambient pressure (Limpijumnog and Lambrecht 2001). At high pressure, several of ionic semiconductors undergo a transition from wurtzite to rocksalt. Therefore, the wurtzite to rocksalt transition via certain possible intermediate phase is of particular interest in this class of semiconductors. Evidently, this becomes of greater interest since the wurtzite to rocksalt phase transition under pressure has been observed for III$\mathrm{V}$ nitrides (AlN, GaN, and InN) (Prinz 1990; Berkowitz et al 1992; Xiao et al 1992; Hayashi et al 1997; Wellmann et al 1997; Tanaka et al 1999; Ohno 2000), and recently the isostructural phase transition (IPT) has been reported in $\mathrm{GaN} / \mathrm{ScN}$ and $\mathrm{InN} / \mathrm{ScN}$ superlattices (Ranjan et al 2005). Up-to-date, none of the experimental or theo- retical studies have predicted the kind of phase transitions that occur in the ( $\mathrm{Sc}, \mathrm{Ga}) \mathrm{N}$ alloys as a function of the inplane strain. Is it a rocksalt vs wurtzite or even an intermediate phase between the two? This makes the wurtzite to rocksalt transition, or vice versa, and the competition for stability between these two structures of special importance. In order to start answering such a question, one should note that $\mathrm{GaN}$, in its equilibrium ground-state, adopts wurtzite phase structure, while $\mathrm{ScN}$ has the newly discovered five-fold coordinated layered structure denoted by $h-\mathrm{ScN}$ (Farrer and Bellaiche 2002). So this raises the question of wurtzite vs $h$-ScN structural bonding preference in this alloy system.

These interesting predictions and findings have encouraged us to go beyond the typical structural equilibrium investigations of the alloy, ScGaN, to study the structural and piezoelectric properties as a function of the in-plane strain. In particular, we focus on $\mathrm{ScGaN}$ materials that are under tensile or compressive in-plane strain. In other words, the lattice constant, $a$, is larger, smaller than the equilibrium lattice constant, $a_{\mathrm{eq}}$ and $\eta=\left(a-a_{\mathrm{eq}}\right) / a_{\mathrm{eq}}$, respectively and $\eta=\left(a_{\mathrm{eq}}-a\right) / a_{\mathrm{eq}}$ characterizes the magnitude of tensile and compressive strains.

In this paper, we present our results on the stability of $\mathrm{Sc}_{0.5} \mathrm{Ga}_{0.5} \mathrm{~N}$ alloys as a function of the tensile and compressive in-plane strain and the effect of any structural phase transition on the electromechanical response of this system. We will focus on $\mathrm{Sc}_{0.5} \mathrm{Ga}_{0.5} \mathrm{~N}$ system which consists of stacking, in a periodic fashion, equal layers of hexagonal $\mathrm{GaN}$ and hexagonal $\mathrm{ScN}$ on top of each other along the [0001] direction that has the same space group, independent of the values of the axial ratio $(c / a)$ and the internal atomic coordinates $(u)$. In particular, we chose $\mathrm{Sc}_{0.5} \mathrm{Ga}_{0.5} \mathrm{~N}$ structures for our study, since for an overall Sc composition of $50 \%$, the $\mathrm{ScGaN}$ system was predicted to exhibit a phase transition from a $h$-derived structure $(c / a \approx 1.2$ and $u \approx 0.5)$ to wurtzite-like structure $(c / a \approx 1.6$ and $u \approx 0.375)$ (Ranjan et al 2005 and references therein). In other words, the composition, $x=0 \cdot 5$, separates two distinct compositional behaviours of the structural parameters in (Sc, Ga) N systems.

The aim of this paper is three-fold. First, to predict and interpret different structural and piezoelectric properties resulting from alloying hexagonal $\mathrm{ScN}$ with hexagonal $\mathrm{GaN}$, as a function of the tensile and compressive inplane strains at fixed Sc and Ga compositions. Second, we want to show that ScGaN alloys under either compressive or tensile in-plane strain exhibits phase transition associated with high electromechanical response. In other words, it can act as a multifunctional material exhibiting the properties of semiconductors as well as those of ferroelectrics. Finally, we hope to provide suggestions on how to grow disordered ScGaN alloys that can be used to produce multi-functional devices.

The organization of the paper is as follows. Section 2 presents the methodology implemented in our calcula- 
tions. In $\S 3$, we report and discuss the structural and piezoelectric properties of this alloy. We conclude our work in $\S 4$.

\section{Methodology}

The alloy, ScGaN, under investigation in the present work has two hexagonal parent compounds (e.g. GaN and $\mathrm{ScN}$ ). Both have the direct Bravais lattice with primitive lattice vectors given by

$$
\begin{aligned}
& \vec{a}_{1}=a\left(\frac{1}{2} \hat{x}-\frac{\sqrt{3}}{2} \hat{y}\right), \\
& \vec{a}_{2}=a\left(\frac{1}{2} \hat{x}+\frac{\sqrt{3}}{2} \hat{y}\right), \\
& \vec{a}_{3}=a \hat{z},
\end{aligned}
$$

where $a$ and $c$ are the in-plane and out-of-plane lattice parameters, respectively, and $c / a$ the axial ratio. The unit vectors along the Cartesian axes are denoted by $\hat{x}, \hat{y}$ and $\hat{z}$. The primitive unit cell for these two binaries contains four atoms: two $\mathrm{N}$ atoms located at $\vec{r}_{1}$ and $\vec{r}_{2}$, and two cations of either Ga or Sc located at $\vec{r}_{3}$ and $\vec{r}_{4}$, with

$$
\begin{aligned}
& \vec{r}_{1}=0, \\
& \vec{r}_{2}=\frac{2}{3} \vec{a}_{1}+\frac{1}{3} \vec{a}_{2}+\frac{1}{2} \vec{a}_{3}, \\
& \vec{r}_{3}=u \vec{a}_{3}, \\
& \vec{r}_{4}=\frac{2}{3} \vec{a}_{1}+\frac{1}{3} \vec{a}_{2}+\left(\frac{1}{2}+u\right) \vec{a}_{3},
\end{aligned}
$$

where $u$ is the dimensionless internal parameter. The three structural parameters, $a, c / a$, and the internal parameter, $u$, determine the atomic coordinates of atoms inside the primitive unit cell and are needed to fully understand the structural properties of the hexagonal parent compounds of the ScGaN alloy. Our previous local-density approximation (LDA) (Hohenberg and Kohn 1964; Kohn and Sham 1965) predicted that, on the one hand, GaN exhibits a four-fold coordinated wurtzite phase, which is associated with the polar $p 6_{3} m c\left(C_{6 \mathrm{v}}\right)$ point group. Its equilibrium structural parameters are $a=3.1565 \AA$ (= 5.965 Bhor), $c / a=1.631$, and $u=0.376$ (Al-Yacoub and Bellaiche 2001). These values agree very well with the previous theoretical (Bellaiche 2002) and experimental findings (Bernardini et al 1997). On the other hand, the other hexagonal binary, $\mathrm{ScN}$, exhibits five-fold coordinated layered structure, which is associated with the nonpolar $p 6_{3} / m c c\left(D_{6 \mathrm{~h}}\right)$ space group and is denoted by $h-\mathrm{ScN}$. We predicted the equilibrium structural parameters of $h$ $\mathrm{ScN}$ to be $a=3.5270 \AA$ (=6.916 Bhor), $c / a \approx 1.207$ and $u=0 \cdot 5$, in excellent agreement with previous LDA calculations (Farrer and Bellaiche 2002; Ranjan et al 2003).
We are not aware of any experimental measurements of the $h$-ScN structural parameters. However, previous theoretical full-potential linearized augmented plane wave (FLAPW) predicted that the in-plane lattice constant of $\mathrm{ScN}$ in its rocksalt structure to be $4.52 \AA$ (Leroux and Gil 1999). The experimental measurements predicted the $a$ parameter of $\mathrm{ScN}$ in its rocksalt structure to be $4.50 \AA$ (Park et al 1984; Lengauer 1988; Gubanov et al 1996; Schilfgaarde et al 1997) and $4.44 \AA$ (Wyckoff 1963). Furthermore, the four-atom unit cell of the (Sc, Ga) $\mathrm{N}$ alloy in the present work consists of stacking different layers along the [0001] direction. For instance, $\mathrm{Sc}_{0.5} \mathrm{Ga}_{0.5} \mathrm{~N}$ alloys consist of stacking equal number of $\mathrm{ScN}$ layers on the top of the same number of GaN layers along $\vec{a}_{3}$ direction. It is important to note that this ordered structure exhibits two kinds of $u$ parameter as indicated by (2). The ones binding $\mathrm{Ga}$ atoms to their nearest neighbour $\mathrm{N}$ atoms along $c$-axis is denoted by $u_{\mathrm{GaN}}$, and those connecting Sc atoms to their closest $\mathrm{N}$ atoms along $a_{3}$ is referred to as $u_{\mathrm{ScN}}$.

Computationally, total-energy calculations are carried out using first-principles density-functional theory (DFT) within LDA (Hohenberg and Kohn 1964; Kohn and Sham 1965), and the Vanderbilt ultrasoft pseudopotentials (Vanderbilt 1990). The semicore states of Sc, Ga and N atoms are all included in the valence states. We used the Ceperley-Alder (1980) exchange-correlation function as parametrized by Perdew and Zunger (1981). A planewave cutoff of 25 Ry was chosen. We also used a $6 \times 6 \times 4$ Monkhorst-Pack (Monkhorst and Pack 1976) for Brillouin-zone integration of the four-atom unit cell of the parents and the resulting ( $\mathrm{Sc}, \mathrm{Ga}) \mathrm{N}$ alloy. The full relaxations of the structural parameters in every structure are followed by the minimization of the total-energy of the unit cell and Hellman-Feynman forces on each atom (these later being smaller than $0.045 \mathrm{meV} / \AA$ at convergence). We also used the modern theory of polarization (Hohenberg and Kohn 1964) and the Berry phase approach of Bloch states to compute the spontaneous polarization, P. The piezoelectric coefficients are derived from knowing $\mathbf{P}$ via (Kohn and Sham 1965)

$$
e_{i j}=\frac{1}{2 \pi \Omega} \sum_{\alpha} R_{\alpha, i} \frac{d}{d \eta_{j}}\left(\Omega \vec{G}_{\alpha} \cdot \vec{P}\right)
$$

where $R_{\alpha, i}$ represents the components of primitive lattice vectors, $\mathbf{R}_{\alpha}$, along the $i$ axis and $\alpha=1,2,3$ is used to index the three real-space primitive lattice vectors, $\mathbf{R}_{\alpha}$. The three reciprocal-space primitive lattice vectors are denoted by $\mathbf{G}_{\alpha}$ and $\eta_{j}$ the applied macroscopic strain and $\Omega=\sqrt{3} a^{2} c / 2$ the volume of the unit cell, where $a$ is the in-plane lattice constant and $c / a$ the axial ratio. Equation (3) is evaluated by computing the spontaneous polarization in two different state configurations: one is that of the initial ground state, and the other is the state after the macroscopic strain, $\eta_{j}$, is applied relative to the initial 

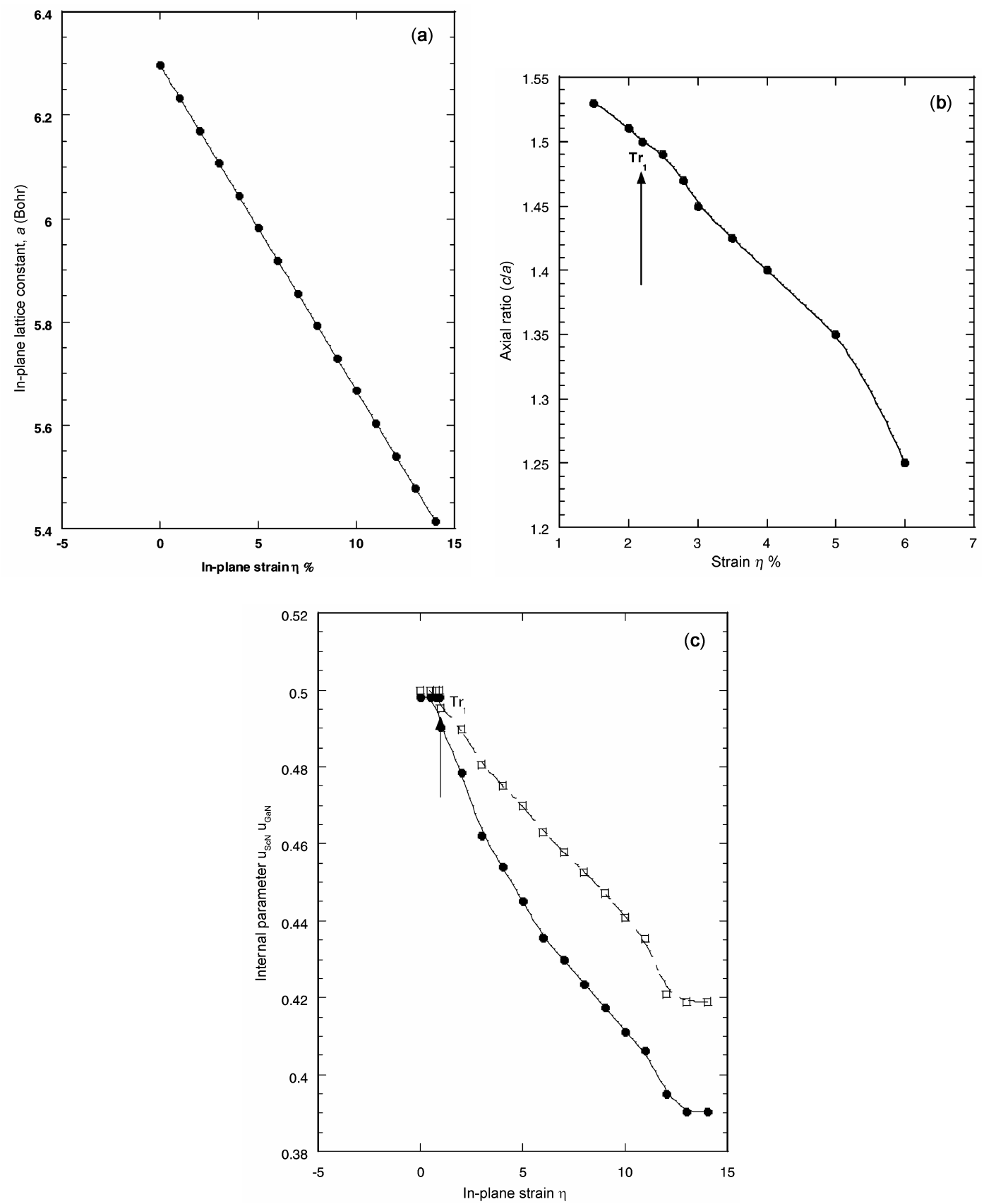

Figure 1. Structural properties of ordered $\mathrm{Sc}_{0.5} \mathrm{Ga}_{0.5} \mathrm{~N}$ alloys as a function of the compressive in-plane strain $\eta$. (a) Variation of the equilibrium in-plane lattice constant as a function of the in-plane compressive strain, $\eta,(\mathbf{b})$ displaying the axial ratio $c / a$. The arrow indicates the region of particular interest discussed in the text and (c) displaying the internal parameters, $u_{\mathrm{ScN}}$ and $u_{\mathrm{GaN}}$ as a function of $\eta$.

ground state. For this latter configuration state, the atomic coordinates are once again relaxed to respond to the applied macroscopic strain by minimizing the total-energy and the forces on each atom. In our calculation of the $e_{i j}$ piezoelectric coefficients, the final value of $e_{i j}$ is averaged out over the ones obtained for positive and negative strains, $\eta_{j}= \pm 1 \cdot 5 \%$. To gain a deeper insight into the piezoelectric behaviour of this interesting system, the $e_{i j}$ is decomposed into the 'clamped-ion' and the 'intrinsic' contributions (Al-Yacoub and Bellaiche 2001; Bellaiche 2002)

$$
e_{33}=e_{33, c}+e_{33, i} \text {. }
$$

The clamped-ion contribution is calculated using (3) with the internal coordinates of the anions and cations kept frozen at their equilibrium positions of the ground-state. The $e_{33, i}$ intrinsic component comes out as a result of the 
relaxation of internal atomic coordinates when the strain is applied, and is usually calculated as the difference between $e_{33}$ and $e_{33, c}$.

\section{Results}

\subsection{Structural properties}

Figures 1(a)-(c) display the equilibrium in-plane lattice constant, $a$, the axial ratio, $c / a$ (per four atoms), and the internal parameters, $u_{\mathrm{GaN}}$ and $u_{\mathrm{ScN}}$, as a function of the inplane compressive strain, $\eta$, of the Sc-based cation-mixed $\mathrm{Sc}_{0.5} \mathrm{Ga}_{0.5} \mathrm{~N}$ ordered alloy. It has been predicted that unstrained $\mathrm{Sc}_{0.5} \mathrm{Ga}_{0.5} \mathrm{~N}$ stabilizes in a wurtzite-derived structure (Ranjan et al 2005). This prediction was in complete agreement with the experimental findings (Ranjan et al 2005 and references therein) that the equilibrium ground state of ( $\mathrm{Sc}, \mathrm{Ga}) \mathrm{N}$ disordered alloys is wurtzite-like for Ga compositions larger than $70 \%$ and nonwurtzite-like for Ga concentrations less than $46 \%$. Experimentally, the nonwurtzite-like phase observed for disordered ( $\mathrm{Sc}, \mathrm{Ga}$ ) $\mathrm{N}$ alloys is rocksalt rather than $h$-derived. This is because the observations of the metastable $h$-derived phase may require special growth techniques other than the available conventional techniques. The $h$-derived phase is easily detected in ordered ( $\mathrm{Sc}, \mathrm{Ga}$ ) $\mathrm{N}$ alloys since it exhibits full planes of $\mathrm{GaN}$ that prefers to crystallize in a wurtzite structure rather than rocksalt phase. These outstanding observations have encouraged us to investigate the beha- viour of this ordered system as a function of in-plane strain, which is experimentally an accessible parameter and at a fixed Sc composition, which is rather experimentally less accessible. Figure 1 indicates that as the inplane compressive strain is increased, the lattice constant, $a$, linearly decreased and the resulting behaviour of $c / a$ and $u$ vs $\eta$ demonstrates the existence of a phase transition, to be indicated by $\operatorname{Tr}_{1}$, occurring for a compressive strain of $2 \%$. This phase transition is associated with the deviation of $u_{\mathrm{GaN}}$ and $u_{\mathrm{ScN}}$ with respect to the equilibrium value of 0.5 , and the deviation of the axial ratio $c / a$ with respect to $1 \cdot 51$. Such a deviation indicates a change of space group from nonpolar $P 6_{3} / m m c$ (i.e. the axial ratio and internal parameters are relatively close to those of $h$ $\mathrm{ScN}$ ) to a polar $P 6_{3} / m c$. This change of phase leads to the fact that the Sc, Ga atoms, on the one hand, and $\mathrm{N}$ atoms, on the other hand, are no longer sitting on the same (0001) plane. Consequently, the $\operatorname{Tr}_{1}$ phase transition results in the appearance of spontaneous electrical polarization in the strained ordered $\mathrm{Sc}_{0.5} \mathrm{Ga}_{0.5} \mathrm{~N}$ alloys, which are close in its magnitude, to the temperature-induced spontaneous electrical polarization in some perovskite compounds (Hohenberg and Kohn 1964). Figure 2 also illustrates the structural parameter behaviour of ordered $\mathrm{Sc}_{0.5} \mathrm{Ga}_{0.5} \mathrm{~N}$ alloys as a function of tensile in-plane strain. The figure displays the resulting behaviour of $u_{\mathrm{ScN}}$ and $u_{\mathrm{GaN}}$ and $c / a$ vs tensile in-plane strain and demonstrates the existence of a phase transition, to be indexed by $\operatorname{Tr}_{1}$, occurring for a tensile strain, $\eta$, close to $6 \cdot 25 \%$. The $\operatorname{Tr}_{1}$ transition is associated with the deviation of $u_{\mathrm{ScN}}$ and $u_{\mathrm{GaN}}$ with respect to the
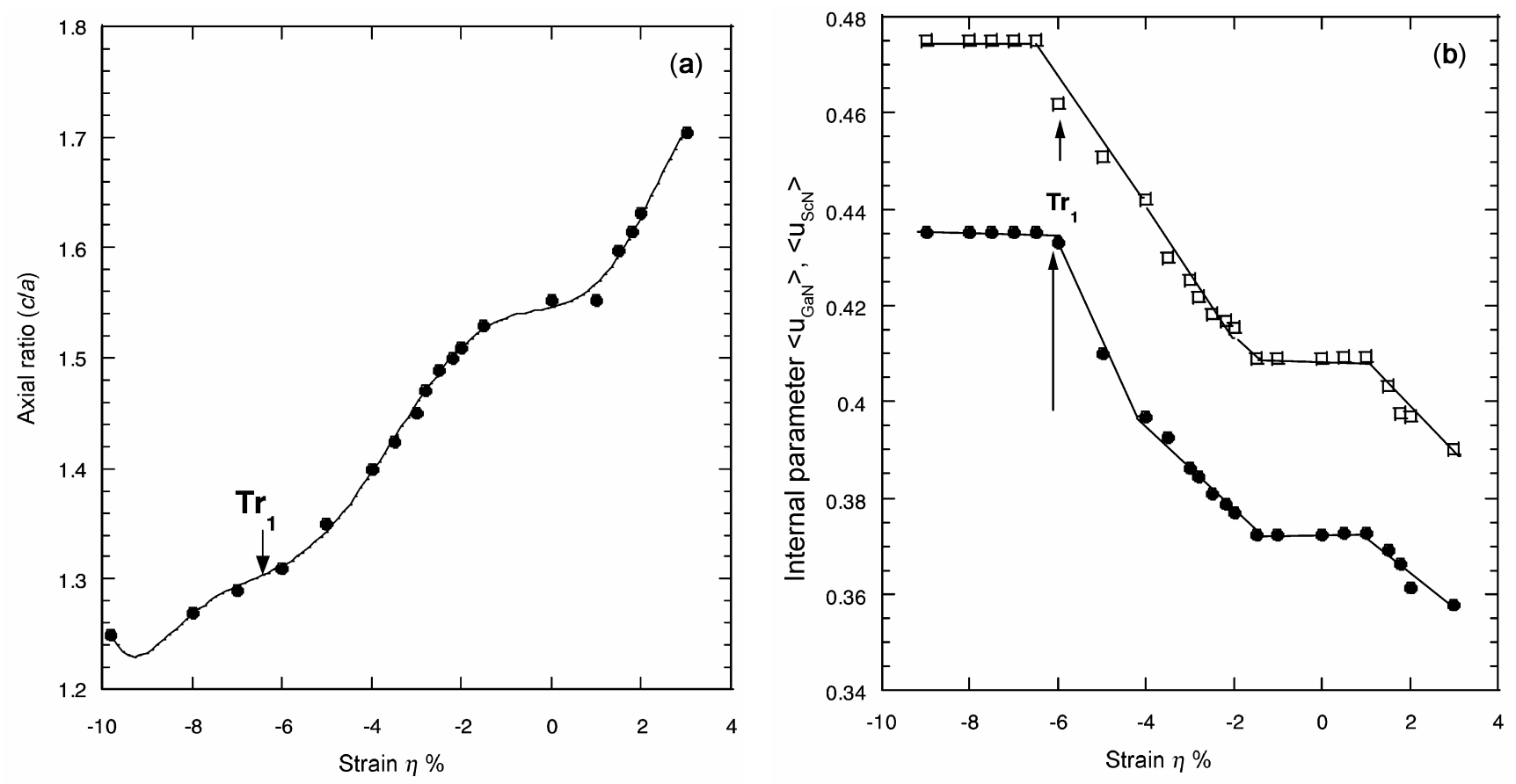

Figure 2. Structural properties of ordered $\mathrm{Sc}_{0.5} \mathrm{Ga}_{0.5} \mathrm{~N}$ alloys as a function of in-plane tensile strain, $\eta$. (a) and (b) display the axial ratio, cla and the $u_{\mathrm{ScN}}$ and $u_{\mathrm{GaN}}$, respectively. The arrows indicate the regions of particular interest discussed in the text. 
equilibrium values of 0.4315 and 0.4715 , respectively, and the deviation of the axial ratio, $c / a$, from the equilibrium value of 1.207 . Such a deviation results in a change of space group from the nonpolar $P 6_{3} / m m c$ to polar $P 6_{3} / m c$. This phase transition leads to the existence of a large spontaneous electrical polarization. The appearance of such a spontaneous polarization enhances the total polarization and results in huge $e_{33}$ piezoelectric coefficients, as explained in the next section.

\subsection{Piezoelectric properties}

Figures 3 and 4 shed some light on the electromechanical properties of the $\mathrm{Sc}_{0.5} \mathrm{Ga}_{0.5} \mathrm{~N}$ by displaying the piezoelectric coefficient, $e_{33}$, as predicted within the modern theory of polarization and Berry phase approach (King-Smith and Vanderbilt 1993; Bellaiche and Vanderbilt 1999), as a function of the compressive and tensile in-plane strain, $\eta$, respectively. One can clearly see that $\operatorname{Tr}_{1}$ leads to the appearance and huge enhancement of piezoelectricity, in consistence with the in-plane strain-induced development of a spontaneous polarization. In particular, our firstprinciples calculation yield $e_{33}$ piezoelectric coefficients of magnitude $8.3 \mathrm{C} / \mathrm{m}^{2}$ which occurs at a compressive inplane strain of $2 \%$, and $6.2 \mathrm{C} / \mathrm{m}^{2}$ which occurs at a tensile in-plane strain of $6 \cdot 25 \%$. Such $e_{33}$ values are considered to be one of the largest piezoelectric coefficients ever predicted using first-principles electronic structure methods (Bellaiche and Vanderbilt 1999; Sai et al 2001). This is remarkable when realizing that the corresponding piezo-

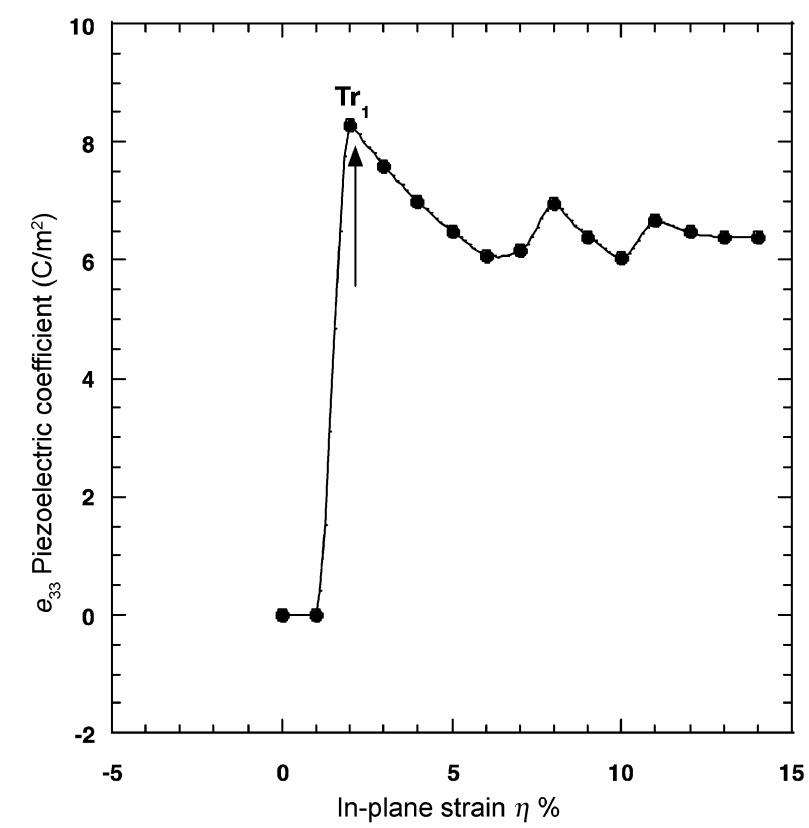

Figure 3. The $e_{33}$ piezoelectric coefficient of ordered $\mathrm{Sc}_{0.5}$ $\mathrm{Ga}_{0.5} \mathrm{~N}$ alloys as a function of compressive in-plane strain, $\eta$. The figure indicates a huge enhancement of piezoelectricity at a compressive strain, $\eta=2 \%$. The arrow indicates the region of particular interest discussed in text. electric coefficients of $\mathrm{PbTiO}_{3}$ and $\mathrm{Pb}(\mathrm{Mg}, \mathrm{Nb}, \mathrm{Ti}) \mathrm{O}_{3}$ compounds are $\approx 4$ and $9 \mathrm{C} / \mathrm{m}^{2}$, respectively (Leroux and Gil 1999; Saghi-Szabo et al 1998). In other words, the ordered $\mathrm{Sc}_{0.5} \mathrm{Ga}_{0.5} \mathrm{~N}$ alloys exhibit piezoelectric coefficients similar in magnitude to that of ferroelectric perovskites, which are the current materials of choice for piezodevices. Furthermore, figures 3 and 4 indicate that any further increase in either compressive or tensile in-plane strain would result in a significant decrease of piezoelectric coefficients. We have demonstrated that longitudinally or compressively strained (Sc, Ga) N can exhibit optimized electromechanical properties by undergoing structural changes. It is now clear that the suggestions of Farrer and Bellaiche (2002) to be correct. As a matter of fact, alloying $h$-ScN with wide band gap wurtzite material (e.g. GaN) should lead to the structural path which involves the evolution from a five-fold coordinated nonpolar structure with a relatively small band gap to a four-fold coordinated polar phase with a much larger band gap, when the Sc concentration is continuously decreased. A paraelectricto-ferroelectric $\operatorname{Tr}_{1}$ transition, yielding large $e_{33}$ piezoelectric coefficients should thus occur along this path for some specific Sc concentrations. Interestingly, variation of the Sc composition in disordered $\mathrm{Sc}_{x} \mathrm{Ga}_{1-x} \mathrm{~N}$ along with varying tensile or compressive in-plane strain should lead to practically grown materials bridging the semiconductors and ferroelectrics, especially when realizing the discovery of several modern growth techniques in the last two decades.

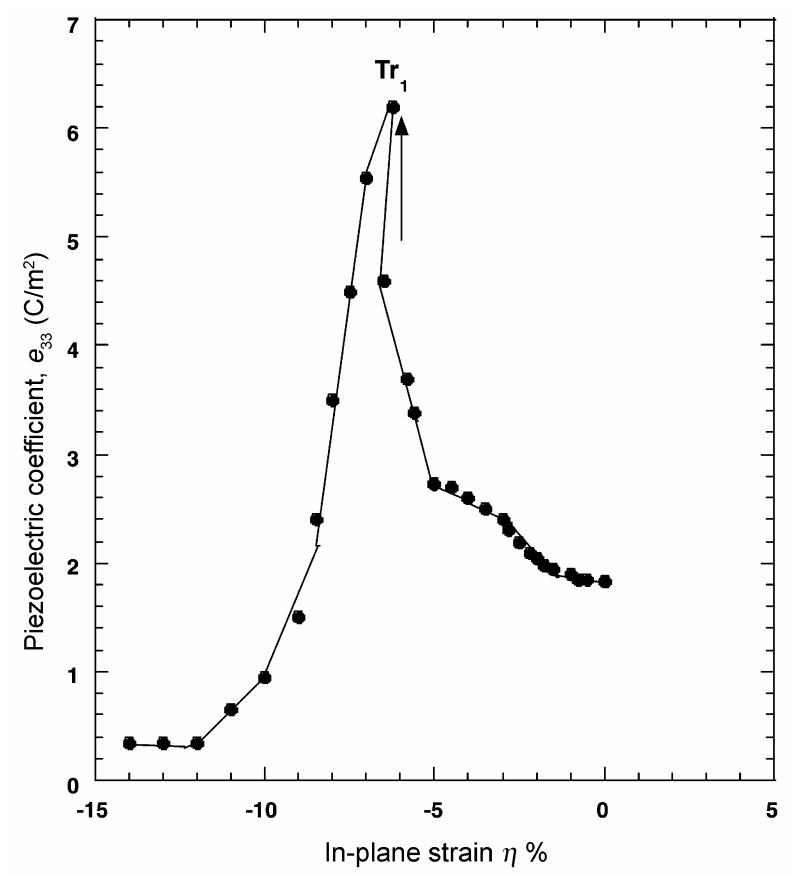

Figure 4. Electromechanical properties of ordered $\mathrm{Sc}_{0.5} \mathrm{Ga}_{0.5} \mathrm{~N}$ alloys as a function of the in-plane strain, $\eta$. It displays the $e_{33}$ piezoelectric coefficient as a function of tensile in-plane strain, $\eta$. It shows a huge enhancement of $e_{33}$ at a tensile strain of $6.25 \%$. 


\section{Conclusions}

In conclusion, we have performed first-principles calculations on ordered $\mathrm{Sc}_{0.5} \mathrm{Ga}_{0.5} \mathrm{~N}$ alloys as a function of inplane compressive and tensile strain, and compared their structural and piezoelectric properties with the corresponding properties of their $\mathrm{GaN}$ and $\mathrm{ScN}$ parent compounds (in their most stable hexagonal forms). It has been demonstrated that transition from hexagonal-derived, which has the structural $c / a$ and $u$ parameters close to that of hexagonal $\mathrm{ScN}$ (i.e. $\approx 1.2$ and $0 \cdot 5$, respectively), to the wurtzite-derived structure, which has the corresponding structural parameters of $\approx 1.6$ and 0.376 , respectively, occurs at an overall Sc composition of $50 \%$. At this specific Sc composition, the ordered $\mathrm{Sc}_{0.5} \mathrm{Ga}_{0.5} \mathrm{~N}$ alloys are also found to have rather distinct structural and piezoelectric properties. In particular, the piezoelectric $e_{33}$ coefficients of these alloys are found to exhibit values that are very close to those of perovskites ferroelectrics. As a result, this material exhibits some structural and piezoelectric properties that are technologically very promising. The realization of several technologically used materials that have attracted much recent attention, such as $(\mathrm{Al}, \mathrm{Ga}$, In)/(Ga, In)N quantum wells and $(\mathrm{Ga}, \mathrm{In}) \mathrm{N}$ quantum dots requires an interplay between composition in the active layers and the strain induced by the substrate on the top of which the active layers are to be grown. Consequently, we would like to suggest that by simultaneous variation of compressive or tensile in-plane strain, and the Sc composition, it is possible to yield very interesting epitaxially grown hexagonal $\mathrm{Sc}_{x} \mathrm{Ga}_{1-x} \mathrm{~N}$ alloys. For instance, one can think of a disordered $\mathrm{Sc}_{x} \mathrm{Ga}_{1-x} \mathrm{~N}$ for which $x$ concentration yields a paraelectric phase very close to the border of the paraelectric-ferroelectric $\operatorname{Tr}_{1}$ transition. Growing of such a material epitaxially on a hexagonal substrate having a slightly smaller or larger in-plane constant could produce a compressive or tensile strain which is able to induce this transition, and thus will lead to an enhancement of the piezoelectricity. As a result, the fabrication of multifunctional smart devices can be accomplished.

\section{Acknowledgements}

We are grateful for the financial assistance provided by the faculty of scientific research at Jordan University of Science and Technology, Grant No. 29/2003. The authors acknowledge E. Sigmund, Brandenburg Technical University in (BTU), Cottbus, Germany, for useful discussions. Computational support has been provided by the Theoretical Physics Institute at BTU, Germany.

\section{References}

Al-Yacoub A and Bellaiche L 2001 Appl. Phys. Lett. 792166 Bellaiche L 2002 Curr. Opin. Solid State Matter. Sci. 619
Bellaiche L and Vanderbilt D 1999 Phys. Rev. Lett. 831347

Berkowitz A E et al 1992 Phys. Rev. Lett. 683745

Bernardini F, Fiorentini V and Vanderbilt D 1997 Phys. Rev. B56 R10024

Ceperley D M and Alder B 1980 J. Phys. Rev. Lett. 45566

Christensen N E and Gorczyca I 1994 Phys. Rev. B50 4397

Constantin C, Al-Brithen H, Haider M B, Ingram D and Smith A R 2004 Phys. Rev. B70 193309

Damilano B et al 1999 Appl. Phys. Lett. 75962

Dismukes J P and Moustakas T D 1996 Proceedings of the III$V$ nitride materials and processes symposium, ECS (Pennington, NJ: The Electrochemical Society) 96-11 p. 111

Edgar J H, Strite S, Akasaki A, Amano H and Wetzel C 1999 Properties, processing and applications of gallium nitride and related semiconductors (London: Inspec) Vol. 23

Farrer N and Bellaiche L 2002 Phys. Rev. B66 201203(R)

Gall D, Stadele M, Jarrendahl K, Petrov I, Desjardins P, Haasch R T, Lee T-Y and Greene J E 2001 Phys. Rev. B63 125119

Gubanov V A, Lu Z W, Klein B M and Fong C Y 1996 Phys. Rev. B53 4377

Hayashi T, Tanaka M, Nishinaga T and Shimada H 1997 J. Appl. Phys. 814865

Hohenberg P and Kohn W 1964 Phys. Rev. 136864

King-Smith R D and Vanderbilt D 1993 Phys. Rev. B47 R1651

Kohn W and Sham L 1965 J. Phys. Rev. 140 A1133

Lambrecht W R L 2000 Phys. Rev. B62 13538

Lengauer W J 1988 Solid State Chem. 76412

Leroux M and Gil B 1999 Gallium nitride and related semiconductors, Emis DATAREVIEWS SERIES 23 (London: INSPEC) p. 45

Limpijumnog S and Lambrecht W R L 2001 Phys. Rev. B63 104103

Monkhorst H J and Pack J D 1976 Phys. Rev. B13 5188

Ohno H 2000 Physica E (Amsterdam) 6702

Park K T, Terakura K and Hamada N 1984 J. Phys. C20 1241

Perdew J P and Zunger A 1981 Phys. Rev. B23 5048

Prinz G A 1990 Science 2501092

Ranjan V, Bellaiche L and Walter E J 2003 Phys. Rev. Lett. 90 257602

Ranjan V, Bin-Omran S, Bellaiche L and Ahmad Alsaad 2005 Phys. Rev. B71 195302

Ranjan V, Bin-Omran S, Sichuga D, Nichlos R S, Bellaiche L and Alsaad A 2005 Phys. Rev. B72 085315

Sai N, Meyer B and Vanderbilt D 2001 in Proceedings of the 11th Williamsburg ferroelectric workshop (ed.) H Krakauer (New York: AIP) p. 218

Saghi-Szabo G, Cohen R E and Krakauer H 1998 Phys. Rev. Lett. 804321

Schilfgaarde M V, Sher A and Chen A B 1997 J. Cryst. Growth 1788

Takeuchi N 2002 Phys. Rev. B65 045204

Tanaka M, Saito K and Nishinaga T 1999 Appl. Phys. Lett. 7464

Travaglini G, Marabelli F, Monnier R, Kaldis E and Wachter P 1986 Phys. Rev. B34 3876

Vanderbilt D 1990 Phys. Rev. B41 R7892

Wellmann P J, Garcia J M, Feng J L and Petroff P M 1997 Appl. Phys. Lett. 712532

Wyckoff R W G 1963 Crystal structures (New York: Wiley) 2nd edn, Vol. 1, p. 86

Xiao Q, Jiang J S and Chien C L 1992 Phys. Rev. Lett. 683749 